\title{
Anisotropic microsphere-based approach to damage in soft fibered tissue
}

\author{
P. Sáez • V. Alastrué · E. Peña • M. Doblaré · M.A. Martínez
}

Received: date / Accepted: date

\begin{abstract}
An anisotropic damage model for soft fibered tissue is presented in this paper, using a multi scale scheme and focusing on the directionally-dependent behavior of these materials. For this purpose, a microstructural or, more precisely, a microsphere-based approach is used to model the contribution of the fibers. The link between micro-structural contribution and macroscopic response is achieved by means of computational homogenization, involving numerical integration over the surface of the unit sphere. In order to deal with the distribution of the fibrils within the fiber, a von Mises probability function is incorporated, and the mechanical (phenomenological) behavior of the fibrils is defined by an exponential-type model. We will restrict ourselves to affine deformations of the network, neglecting any cross-link between fibrils and sliding between fibers and the surrounding ground matrix. Damage in the fiber bundles is introduced through a thermodynamic formulation, which is directly included in the hyperelastic model. When the fibers are stretched far from their natural state, they become damaged. The damage increases gradually due to the progressive failure of the fibrils that make up such a structure. This model has been implemented in a finite element code, and different boundary value problems are solved and
\end{abstract}

P. Sáez · E. Peña · M. Doblaré · M.A. Martínez

Group of Structural Mechanics and Materials Modeling.

Aragón Institute of Engineering Research. University of Zaragoza.

CIBER-BBN. Centro de Investigación Biomédica en Red en Bioingeniería, Biomateriales y Nanomedicina.

E-mail: psaez@unizar.es

V. Alastrué

EBERS Medical Technology SL.

Av. de Madrid, 117, 5A, E-50010, Zaragoza, SPAIN discussed herein in order to test the model features. Finally a clinical application with the material behavior obtained from actual experimental data is also presented.

Keywords Soft tissue - Microsphere · Affine deformations $\cdot$ Damage $\cdot$ Blood vessels

\section{Introduction}

Most biological soft tissues, and particularly blood vessels, are composed of networks of collagen fiber bundles (Rhodin, 1980) embedded in an almost isotropic ground substance with a high water content, which provides a well known quasi-incompressible behavior (Carew et al., 1968; Chuong and Fung, 1984). These collagen bundles are made up of fibrils and these, in turn, are composed of aggregates of collagen molecules. Blood vessels have three main layers, intima, media and adventitia (see, e.g., Fung (1990)). The media is mainly composed of smooth muscle cells and sheets of collagen fibers oriented preferentially along the circumferential direction. The adventitia is basically composed of a more random distribution of collagen fiber bundles while the intima is a thin layer of endothelial cells. The orientation of the collagen fibers is mainly responsible for the anisotropic response and the highly non-linear behavior of blood vessels, as described in numerous works (see, e.g., Fung (1990) and Humphrey (1995)). This structure at the micro-level is of crucial importance in the macroscopic behavior and the characterization of these materials, as we will discuss later.

Many constitutive models have been proposed lasting recent years to model these biological tissues within the incompressible or quasi-incompressible hyperelastic framework. In this context, the description of a given 
material is related to the definition of a strain energy density function (SEDF) from which all the mechanical relations and variables can be obtained. Early SEDFs in soft tissue mechanics were purely phenomenological, whereby non micro-structural information was gathered, so they were able to describe only some aspects of the material behavior, usually only under physiological loads. However, many of them have been widely used for this purpose with satisfactory results (Demiray et al., 1988). In order to provide a more realistic characterization of the tissue, structural models, in which structural tensors are introduced in the free energy function to take into account the anisotropy of the material, have been proposed to deal with these limitations (see, e.g., Boehler (1987); Holzapfel et al. (2000); Menzel and Steinmann (2003); Holzapfel et al. (2005)). Although these represented a clear advance in the mechanical characterization of such materials, many of the more recent works have tried to advance further towards a more detailed micro-structural experimental characterization which has allowed improved micro-structural models. For example, the works by Taber (1998), Humphrey and Rajagopal (2002) and Ateshian (2007) are related to the growth of micro constituents, while Kuhl and Holzapfel (2007) and Himpel et al. (2008) presented some results on the remodeling of collagen and elastin. Moreover, Gasser et al. (2006) and later Menzel et al. (2008) included micro-structural information in the hyperelastic formulation through the assumption of a statistical distribution of the fiber orientation around a preferential direction.

The use of these models is usually limited to the range of physiological loads remaining in the range of the elastic region. Most finite element implementations carried out have been limited to this region and only a few have dealt with soft tissue failure. Some pathological processes, such as aneurysms or over-stretched tendon, or clinical surgeries such as angioplasties or clamping lead to material damage. Damage in soft biological tissue is produced by the progressive failure or softening of either the matrix or the fibers. In this context, some authors have proposed continuum damage models for only one component of the material (fibers or matrix), applied to transversally anisotropic soft tissues following the approach of Simo (1987) (Natali et al., 2005; Balzani et al., 2006; Calvo et al., 2007; Ehret and Itskov, 2009; Peña and Doblaré, 2009), or that of Göktepe and Miehe (2005) and Dal and Kaliske (2009) in connection the microsphere framework for failure of rubber-like materials. Other models for the failure of fibrils (see e.g Buehler (2008); Tang et al. (2010)) use molecular simulations to provides a more detailed insight into the failure process of collagen fibrils, which is basically due to the rupture of the cross-links between the tropocollagen molecules that compose the fibril. In the framework of cytoskeletal and polymers networks, Head et al. (2003) presented a different regime of elastic response (affine and non affine) depending of the quality and density of the filament links, leading to an affine response under an unlinked scenario. This is the situation where our model is placed. Future works will take into account other important features.

In short, the high complexity of biological tissues requires mechanical models that include information of the underlying constituents and look at the processes occurring within the material. This behavior of the micro-constituents can be incorporated into macroscopic models by means of computational homogenization. It is in this context where the microsphere-based approach acquires considerable relevance. Miehe et al. (2004), Miehe and Göktepe (2005) and Göktepe and Miehe (2005) used the microsphere approach with emphasis on elastomers. Caner and Carol (2006) were the first to apply this approach to soft biological tissues. Later Alastrué et al. (2009a) focused on the anisotropy of the material.

In this work, we present a three-dimensional decoupled finite strain formulation with a multi scale model for the anisotropic part. We make use of a continuum damage model through the classical $[1-D]$ theory. The damage model is apply to the collagen fibers since damage along the fibers has been shown to be the most important issue. The spatial organization of the collagen fiber bundle, which is made up of collagen fibrils placed around a preferential direction, is taken into account by means of a von Mises statistical distribution function. With this approach, a more realistic response of the damage evolution is expected due to the evolution of the damage from fibril to fibril that make up the fiber bundle. We have limited ourself to an affine model, without taking into account the existing crosslinks between fibrils or the sliding between fibers and matrix.

The paper is organized as follows: Section 2 describes the formulation of the material model, including the microsphere approach, damage model and the mechanical behavior. Section 2.1 presents the essential kinematics while Section 2.2 deals with the splitting of the strain energy density function (SEDF) into volumetric and deviatoric parts, which can be split further into isotropic and anisotropic parts. Next, the microsphere model is reviewed in Section 2.3. Section 2.4 highlights the mechanical response for both the matrix and fibers, while Section 2.5 discusses the da- mage model and particularizes it to fiber damage under the microsphere based approach. Section 3 presents a sen- 
sitivity analysis of different parameters in different geometries. Section 4 shows the results of an example of clinical interest and, finally, section 5 presents the main conclusions of this work.

\section{Material model}

\subsection{Kinematics}

Let $\Omega_{0}$ be the reference or material configuration of a continuum body $\mathcal{B}$ and $\Omega$ the current or spatial configuration at time $t$, regarding an arbitrary reference system (Cartesian from now on). Let $\mathbf{X} \in \Omega_{0}$ be the position of a particle in the reference configuration and $\mathbf{x} \in \Omega$ the position of the same particle at time $t$. The motion mapping $\varphi: \mathbf{X} \rightarrow \mathrm{x}$ relates the position of $\mathbf{x}$ to $\mathbf{X}, \mathbf{x}=\boldsymbol{\varphi}(\mathbf{X})$. The gradient of $\boldsymbol{\varphi}$ with respect to $\mathbf{X}, \mathbf{F}=\nabla_{\mathbf{X}} \boldsymbol{\varphi}$, is known as the deformation gradient tensor. The determinant of deformation gradient, $J$, is given by $J=\operatorname{det}(\mathbf{F})$.

In order to reproduce the quasi-incompressible behavior of soft tissues, an uncoupled representation of the SEDF is used (Flory, 1961). The multiplicative decomposition of the deformation gradient and the CauchyGreen tensor $\mathbf{C}=\mathbf{F}^{T} \cdot \mathbf{F}$ can be expressed as

$\mathbf{F}=\left[J^{1 / 3} \mathbf{I}\right] \cdot \overline{\mathbf{F}}$,

$\mathbf{C}=\left[J^{2 / 3} \mathbf{I}\right] \cdot \overline{\mathbf{C}}$,

where the terms $J^{1 / 3} \mathbf{I}$ and $J^{2 / 3} \mathbf{I}$, with $\mathbf{I}$ being the second order unit tensor, are associated with changes of volume, while $\overline{\mathbf{F}}$ and $\overline{\mathbf{C}}$ are the isochoric deformation gradient and the isochoric Cauchy-Green tensor, respectively.

Furthermore, let $\mathbf{r}$ be a vector in the reference configuration. The so called push-forward operator related to the isochoric part $\overline{\mathbf{F}}$, maps this vector field in $\overline{\mathbf{t}} \in \Omega$, in the deformed configuration

$\overline{\mathbf{t}}=\overline{\mathbf{F}} \cdot \mathbf{r}=J^{-1 / 3} \mathbf{t} \quad$ with $\quad\|\overline{\mathbf{t}}\|=\bar{\lambda}=J^{-1 / 3}\|\mathbf{t}\|$,

where $\overline{\mathbf{t}}$ represents the isochoric push-forward of the material vector $\mathbf{r}$ and $\bar{\lambda}$ the isochoric stretch in the direction of $\mathbf{r}$.

\subsection{Hyperelastic framework}

The free energy density function is given by a scalarvalued function $\Psi$ defined per unit reference volume in the reference configuration and for isothermal processes. Flory (1961) postulated the additive decoupled representation of this SEDF in volumetric and isochoric parts as

$\Psi=\Psi_{\mathrm{vol}}+\Psi_{\mathrm{ich}}$.

As discussed in the introduction, soft biological tissues are highly non-linear anisotropic materials. To differentiate between the isotropic and the anisotropic parts, the free energy density function can be split again as

$\Psi=\Psi_{\mathrm{vol}}+\Psi_{\text {iso }}+\Psi_{\text {ani }}$,

where $\Psi_{\text {vol }}$ describes the free energy associated to changes of volume, $\Psi_{\text {iso }}$ is the isochoric isotropic contribution of the free energy (usually associated to the ground matrix) and $\Psi_{\text {ani }}$ takes into account the isochoric anisotropic contribution (associated to the fibers). These equations are often written in terms of invariants $I_{1}, I_{2}$ and $I_{3}$ (first, second and third invariant respectively) for the isotropic part and $\bar{I}_{4}$ and $\bar{I}_{6}$ for the anisotropic part (see e.g Spencer (1971) and Holzapfel (2000)).

The stress and elasticity tensors can be obtained from the strain energy function by simply deriving it with respect to $\mathbf{F}$ once and twice respectively (see Menzel and Steinmann (2003) and Truesdell and Noll (2004) for a complete review of anisotropic hyperelasticity).

Taking the free energy density as a single function of $\mathbf{F}, \Psi(\mathbf{F})$ and under the premise that $\Psi(\mathbf{F})$ is an objective function, it is possible to write $\Psi(\mathbf{F})$ in terms of $\mathbf{C}, \Psi(\mathbf{C})$ which leads to the classical expression of stress tensors (Holzapfel, 2000) as

$\boldsymbol{\tau}=\mathbf{F} \cdot\left[\mathbf{S}_{\mathrm{vol}}+\mathbf{S}_{\mathrm{ich}}\right] \cdot \mathbf{F}^{T}=\boldsymbol{\tau}_{\mathrm{vol}}+\boldsymbol{\tau}_{\mathrm{ich}}$,

where $\mathbf{S}_{\mathrm{vol}}$ and $\mathbf{S}_{\mathrm{ich}}$ are the volumetric and isochoric part of the second Piola-Kirchhoff stress tensor respectively and $\boldsymbol{\tau}_{\text {vol }}$ and $\boldsymbol{\tau}_{\text {ich }}$ the volumetric and isochoric Kirchhoff stress tensors.

In a similar way, the elasticity tensor in the spatial configuration, defined as the push-forward of the material elasticity tensor $\mathbf{C}=\mathbf{C}_{\mathrm{vol}}+\mathbf{C}_{\mathrm{ich}}$, multiplied by $J^{-1}$, is

$\mathbf{c}=J^{-1} \chi_{*}(\mathbf{C})$,

with $\mathbf{C}_{\mathrm{vol}}$ and $\mathbf{C}_{\mathrm{ich}}$ the volumetric and isochoric parts of $\mathbf{C}$ and $\chi_{*}(\bullet)$ the push-forward operator (Marsden and Hughes, 1994).

\subsection{Microsphere based model}

In recent years the most widely used approach for modeling anisotropy in soft tissues has been representing fiber directions by means of an invariant formulation. Lately, the use of statistical distributions has increased, 
and is also adopted in the present work. Furthermore, a microsphere-based approach has been used at a micro scale level. The microsphere approach tries to capture micro-structural information and transfer it into the macroscopic behavior via a homogenization scheme over the unit sphere $\mathbb{U}^{2}$. In this approach, $\mathbb{U}^{2}$ is integrated by $m$ integration directions $\left\{\mathbf{r}^{i}\right\}_{i=1 \ldots m}$ that are weighted by factors $\left\{w^{i}\right\}_{i=1 \ldots m}$, where $\langle\mathbf{r}\rangle \approx \sum_{i=1}^{m} w^{i} \mathbf{r}^{i}=$ 0 and $\langle\mathbf{r} \otimes \mathbf{r}\rangle \approx \sum_{i=1}^{m} w^{i} \mathbf{r}^{i} \otimes \mathbf{r}^{i}=\frac{1}{3} \mathbf{I}$. So an integral over the unit sphere $\mathbb{U}^{2}$ can be approximated by

$$
\langle(\bullet)\rangle=\frac{1}{4 \pi} \int_{\mathbb{U}^{2}}(\bullet) \mathrm{dA} \approx \sum_{i=1}^{m} w^{i}(\bullet)^{i} .
$$

The term $4 \pi$ is a normalization factor, result of the surface integral $\int_{0}^{\theta} \int_{0}^{\phi} \sin (\theta) \mathrm{d} \theta \mathrm{d} \phi$ over the unit sphere. The unit vectors can be expressed in terms of the spherical coordinates $\theta \in[0, \pi)$ and $\phi \in[0,2 \pi)$ as $\mathbf{r}=$ $\sin (\theta) \cos (\phi) \mathbf{e}_{x}+\sin (\theta) \sin (\phi) \mathbf{e}_{y}+\cos (\phi) \mathbf{e}_{z}$ with $\left\{\mathbf{e}_{x}, \mathbf{e}_{y}, \mathbf{e}_{z}\right\}$ the reference Cartesian system. Previous works (Bažant and Oh, 1986; Alastrué et al., 2009a; Ehret et al., 2010) have used used different schemes and compared different number of integration directions for isotropic and anisotropic functions and, in view of the results therein, 368 directions (Heo and $\mathrm{Xu}, 2001$ ) will be used in all the problems simulated in this work. This has been demonstrated to provide sufficiently accurate results for relatively highly anisotropic materials (see Alastrué et al. (2009a)).

As described above, the anisotropic part of the SEDF is related to the fibers in the material. In general, the anisotropic part of the SEDF can be expressed as

$\Psi_{\mathrm{ani}}=\frac{1}{4 \pi} \int_{\mathbb{U}^{2}} n \rho \psi d A$,

where $n$ is the chain density, $\rho$ a statistical value associated with the fibrils dispersion and $\psi$ the free energy density function of the fibril. Note that to include more than one family of fibers the above SEDF is modified as follow

$\Psi_{\text {ani }}=\sum_{j=1}^{N}\left[\frac{1}{4 \pi} \int_{\mathbb{U}^{2}} n \rho_{\mathrm{j}} \psi_{\mathrm{j}} d A\right]_{\mathrm{j}}$

with $\rho_{\mathrm{j}}$ and $\psi_{\mathrm{j}}$ being the distribution function and the SEDF of the $\mathrm{j}$-nth family. The following equations will be given for one family only for the sake of clarity. We will adopt an affine assumption (compare Miehe et al. (2004)). Since an analytical integration of (9) is in general not possible, a discretization of this equation is used

$\Psi_{\mathrm{ani}} \approx \sum_{i=1}^{m} n \rho_{i} w^{i} \psi\left(\bar{\lambda}^{i}\right)$, where $\bar{\lambda}^{i}$ and $\psi\left(\bar{\lambda}^{i}\right)$ are the stretch and the free energy density function associated to each integration direction

The equations for the Kirchhoff stress and the elasticity tensors in the spatial configuration are expressed as

$$
\begin{aligned}
\overline{\boldsymbol{\tau}}_{\mathrm{ani}} & =\sum_{i=1}^{m}\left[n \rho_{i} \psi_{i}^{\prime} \bar{\lambda}^{i^{-1}} \overline{\mathbf{t}}^{i} \otimes \overline{\mathbf{t}}^{i}\right] w^{i}, \\
\overline{\mathbf{c}}_{\mathrm{ani}} & =\sum_{i=1}^{m}\left[n \rho_{i}\left[\psi_{i}^{\prime \prime}-\psi_{i}^{\prime} \bar{\lambda}^{i-1}\right] \bar{\lambda}^{i-2} \overline{\mathbf{t}}^{i} \otimes \overline{\mathbf{t}}^{i} \otimes \overline{\mathbf{t}}^{i} \otimes \overline{\mathbf{t}}^{i}\right] w^{i},
\end{aligned}
$$

where $\psi_{i}^{\prime}$ and $\psi_{i}^{\prime \prime}$ are the first and second derivative of the fibril energy function with respect to $\bar{\lambda}^{i}$.

\subsection{Material behavior}

The definition of a given material in the hyperelastic framework is associated therefore to establishing a free energy density function for each part of the split discussed above. Here, we have used

$\Psi=\Psi_{\mathrm{vol}}(J)+\Psi_{\text {iso }}\left(\bar{I}_{1}\right)+\Psi_{\mathrm{ani}}(n, \rho, \bar{\lambda})$,

with $\bar{I}_{1}=\mathbf{I}: \overline{\mathbf{C}}$ the first strain modified invariant of $\overline{\mathbf{C}}$ and,

$\Psi_{\mathrm{vol}}(J)=\frac{1}{k} \ln ^{2}(\mathrm{~J})$,

$\Psi_{\text {iso }}\left(\bar{I}_{1}\right)=\mu\left[\bar{I}_{1}-3\right]$ with $\mu \geq 0$ and

$\Psi_{\text {ani }}(n, \rho, \bar{\lambda})=\langle n \rho \psi\rangle$.

The matrix is known to be composed of a significant water content, which results in an almost incompressible behavior, so the volumetric part of the energy density function enforces the quasi-incompressibility constraint depending on the value of the penalty parameter $k$ (15). The matrix contributes to the overall behavior through the volumetric and the isotropic parts of the energy density function (16).

Regarding the anisotropic part of the model (17), a statistical distribution of the fibrils around a preferential orientation is considered through a von Mises statistical function. This orientation distribution function is denoted by $\rho$ and has some interesting properties such as symmetry $\rho(\mathbf{r} ; \mathbf{a})=\rho(-\mathbf{r} ; \mathbf{a})$ and rotational symmetry with respect to the preferred orientation $\mathbf{a}$, and can be expressed as $\rho(\mathbf{Q} \cdot \mathbf{r} ; \mathbf{a})=\rho(\mathbf{r} ; \mathbf{a}) \forall \mathbf{Q} \in \mathrm{SO}(3)$. Note that a could be oriented in any direction of the space leading to a mismatch angle $\theta=\arccos (\mathbf{r} \cdot \mathbf{a})$. A $\pi$ periodic von Mises orientation density function (ODF) (18) has been adopted in this work to take into account the fibrils dispersion (Alastrué et al., 2009a)

$\rho(\theta)=4 \sqrt{\frac{b}{2 \pi}} \frac{\exp (b[\cos (2 \theta)+1])}{\operatorname{erfi}(\sqrt{2 b})}$, 


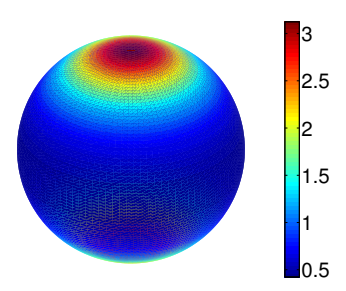

(a) $b=1$.

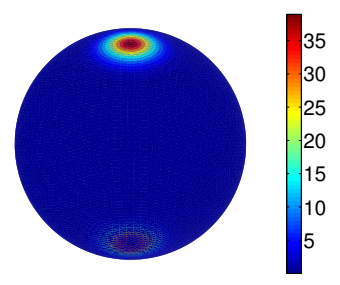

(b) $b=10$.
Fig. 1: Shape of the von Mises ODF for $b=1$ and $b=10$.

where the concentration parameter $b \in \mathbb{R}^{+}$is a measure of the anisotropy. $b \rightarrow 0$ represents an isotropic material, and $b \rightarrow \infty$ a transversally isotropic one. $\operatorname{Erfi}(x)$ is the imaginary error function approximated by a sufficiently large number of terms within its MacLaurin series expansion, which can be written as

$\operatorname{erfi}(x) \approx \pi^{-1 / 2}\left[2 x+\frac{2 x^{3}}{3}+\sum_{j=3}^{k} \frac{x^{2 j-1}}{a(j)}\right]$

where $a(j)=0.5[2 j-1][j-1]$ ! provides a 60 term expansion (Weisstein, 2004), sufficiently accurate for values of $b \leq 20$. Fig. 1 shows the spherical representation of two distributions for different values of $b$.

The contribution of each single collagen fibril in the micro scale is herein assumed as a first approach, to be defined by an exponential-type function, widely used in macroscopic approaches (Holzapfel et al., 2000). In Alastrué et al. (2009a) a comparison between this phenomenological function and the worm-like chain model in the microsphere framework is discussed. Note that, although the integration directions are mathematically identified with the numerical integration scheme, they can also be physically associated to the contribution of the fibrils around each integration direction along its related area. The free density energy associated to each fibril or, equivalently, to each integration direction, is assumed as

$n \psi\left(\bar{\lambda}^{i}\right)= \begin{cases}0, & \text { if } \bar{\lambda}^{i}<1 \\ \frac{k_{1}}{2 k_{2}}\left[\exp \left(k_{2}\left[\left[\bar{\lambda}^{i}\right]^{2}-1\right]^{2}\right)\right] & \text { if } \bar{\lambda}^{i} \geq 1\end{cases}$

with $k_{1} \geq 0$ and $k_{2} \geq 0$ material parameters.

\subsection{Damage model}

The damage model used in this work is able to describe the initial softening effect of the material in a large strain non-linear framework. To deal with behaviors closer to fracture, other techniques such as cohesive models should be used. However, this latter phenomenon is outside the scope of the present work. The adopted damage model uses the concept of internal variables, which provides a general description of materials involving irreversible effects and, therefore, must fulfill the Clausius-Planck inequality. We refer, e.g., to Holzapfel (2000) or Calvo et al. (2007), where this damage formulation is adopted. In its material form and without thermal effects, it can be expressed as $\mathcal{D}=$ $-\dot{\Psi}+\mathbf{P}: \dot{\mathbf{F}} \geq 0$ where $\mathcal{D}$ is the internal dissipation and

$(\bullet)$ is the material time derivative.

Since the volumetric part is related with the water content in soft biological tissue, we shall assume that damage affects only the isochoric part. Moreover, we will restrict ourselves to damage on the anisotropic contribution of the isochoric part. We refer to Holzapfel (2000) and Calvo et al. (2007) for the damage formulation of the isotropic part. Using the previous split form of the free energy, we postulate therefore

$\Psi_{\mathrm{ani}} \approx \sum_{i=1}^{m} n \rho_{i} w^{i} \psi\left(\bar{\lambda}^{i}\right) \approx \sum_{i=1}^{m} n \rho_{i} w^{i}\left[1-D_{i}\right] \psi_{0}\left(\bar{\lambda}^{i}\right)$,

with $D_{i} \in[0,1]$ the monotonically increasing damage internal variables $($ Simo, 1987) for each integration direction and $\psi_{0}\left(\bar{\lambda}^{i}\right)$ the effective strain energy density functions in each integration direction (Eqs. (17)).

In order to fulfill the Clausius-Planck inequality, the internal dissipation $\mathcal{D}_{\text {int }}^{i}=\psi_{0}\left(\bar{\lambda}^{i}\right) \dot{D}_{i} \geq 0$ must be satisfied for $\mathrm{i}=1 \ldots \mathrm{m}$.

$f_{i}=-\partial \psi\left(\bar{\lambda}^{i}\right) / \partial D_{i}=\psi_{0}\left(\bar{\lambda}^{i}\right) \geq 0$

is the thermodynamic driving force associated to damage in each integration direction. The thermodynamic force $f_{i}$ is conjugated to the internal variable $D_{i}$, so the process could be controled by $f_{i}$ instead of $D_{i}$ (see e.g. Simo (1987); Calvo et al. (2007)). The damage evolution equations are given in the strain space; therefore, the free energy is given just by $\bar{\lambda}^{i}$. The damage variable $D_{i}$ is given by the damage energy release rate $\Xi_{i}=\sqrt{2 \psi_{0}\left(\bar{\lambda}^{i}(s)\right)}$ where $\bar{\lambda}^{i}(s)$ are the modified stretches of each integration direction at pseudo-time $s \in \mathbb{R}$.

$\Xi_{i}^{*}=\max _{s \epsilon(-\infty, t]}\left(\sqrt{2 \psi_{0}\left(\bar{\lambda}^{i}(s)\right)}\right)$

is the maximum thermodynamic force achieved along the whole load history. Then, the damage criterion in the strain space is given by the condition

$\Phi_{i}\left(\bar{\lambda}^{i}(s), \Xi_{i}^{*}\right)=\sqrt{2 \psi_{0}\left(\bar{\lambda}^{i}(s)\right)}-\Xi_{i}^{*} \leq 0$, 
which leads to two different situations. If $\Phi_{i}<0$, no damage occurs while $\Phi_{i}=0$ defines the damage surface. Note that $\Phi_{i}>0$ is an impossible situation. An update of this surface is needed when the free energy density of a material point or fibril goes up over $\Xi_{i}^{*}$. Defining the normal components of the damage surface in that space as $N_{i}=\partial_{\bar{\lambda}_{i}} \Phi_{i}$, the following situations may occur:

$\Phi_{i}=0$ and $\left\{\begin{array}{l}N_{i} \dot{\bar{\lambda}}^{i}<0 \\ N_{i} \dot{\bar{\lambda}}^{i}=0 \\ N_{i} \dot{\bar{\lambda}}^{i}>0\end{array}\right.$

which correspond to unloading, neutral loading and loading states, respectively (Simo and Hughes, 1998). The last equation needed for a complete definition of the model is the irreversible rate of the damage variable $D_{i}$

$\frac{\mathrm{d} D_{i}}{\mathrm{~d} t}=\left\{\begin{array}{c}h_{i}\left(\Xi_{i}\right) \dot{\Xi}_{i} \text { if } \Phi_{i}=0 \text { and } N_{i}: \dot{\bar{\lambda}}^{i}>0 \\ 0 \text { otherwise }\end{array}\right.$

where $h_{i}\left(\Xi_{i}\right)=\mathrm{d} D_{i} / \mathrm{d} \Xi_{i}$ are the function that characterize the damage evolution in the material. Finally, the evolution of the maximum thermodynamic force is given as follows

$\dot{\Xi}_{i}{ }^{*}=\left\{\begin{array}{c}\dot{f}_{i}=\partial_{\bar{\lambda}^{i}} \psi_{0} \frac{\dot{\bar{\lambda}}^{i}}{2} \text { if } \Phi=0 \text { and } \dot{f}_{i}>0 \\ 0 \text { otherwise }\end{array}\right.$

The reduction factor is defined as $g_{i}=\left[1-D_{\mathrm{i}}\right]$ and particularized in this work to a sigmoidal function,

$g_{i}=\frac{1}{1+\exp \left(a_{\mathrm{i}}\left[\Xi_{\mathrm{i}}-c_{\mathrm{i}}\right]\right)}$,

where the parameter $a_{i}$ controls the slope and $c_{i}$ defines the value $\Xi_{i}$ such that $g_{i}\left(\Xi_{i}\right)=0.5$ (Fig. 2). Note that we consider the damage parameters $a_{i}$ and $c_{i}$ equal for every integration direction.

The Kirchoff stress tensor $\boldsymbol{\tau}$ and the spatial tangent operator, can be expressed for the anisotropic part as

$\boldsymbol{\tau}_{\mathrm{ani}}=\sum_{i=1}^{m} g_{i} w_{i} n \rho_{i} \psi_{i}^{\prime} \bar{\lambda}^{i-1} \overline{\mathbf{t}}^{i} \otimes \overline{\mathbf{t}}^{i}$

$$
\begin{gathered}
\mathbf{c}_{\mathrm{ani}}=\sum_{i=1}^{m} n \rho_{j} w_{i}\left[g_{i} \psi_{i}^{\prime \prime}-g_{i} \psi_{i}^{\prime} \bar{\lambda}^{i-1}\right. \\
\left.+g_{i}^{\prime} \psi_{i}^{\prime 2}\right] \bar{\lambda}^{i-2} \overline{\mathbf{t}}^{i} \otimes \overline{\mathbf{t}}^{i} \otimes \overline{\mathbf{t}}^{i} \otimes \overline{\mathbf{t}} \mathbb{R},
\end{gathered}
$$

where $\boldsymbol{\tau}_{\text {ani }}$ and $\mathbf{c}_{\text {ani }}$ are the above mentioned anisotropic stress and elasticity tensors. In Algorithm 1 the steps followed to formulate the affine-stretch model with damage are summarized.

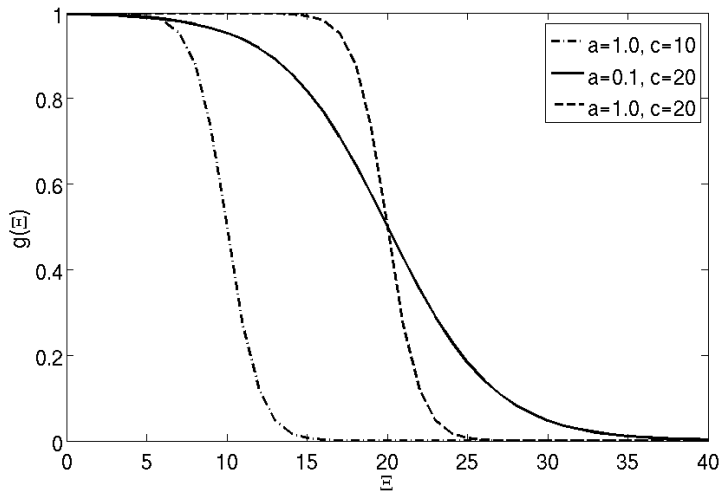

Fig. 2: Representation of the reduction factor $g_{i}$ for different parameters.

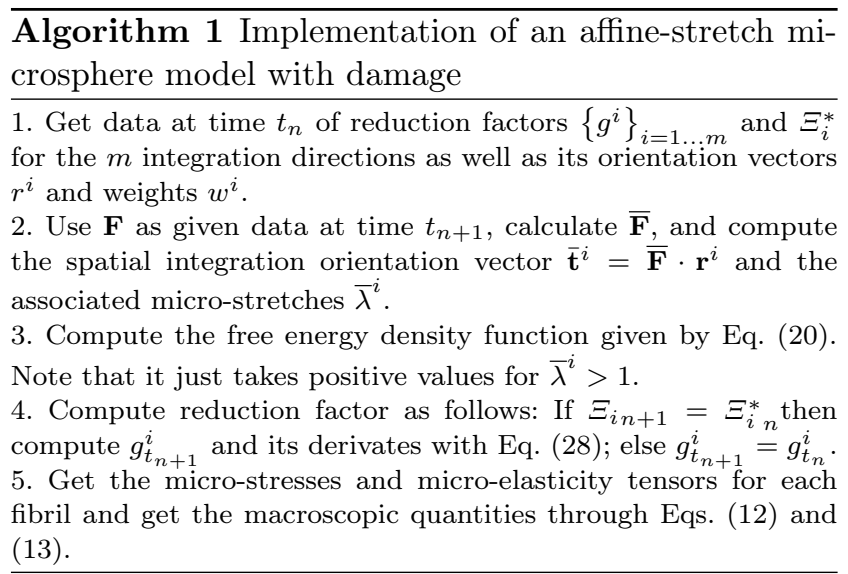

\section{Numerical examples}

The principal aim of this section is to illustrate the performance and the physical mechanics involved in the above presented model. Some simulations are carried out to show the behavior of the model in different situations. A homogeneous deformation state and a thin holed plate have been investigated. The examples have been implemented in the finite element code ABAQUS by means of a UMAT user subroutine. The developed sensitivity analysis responds to the knowledge that several material parameters can be found for different soft tissues and blood vessels in particular.

\subsection{Micro mechanics of the tissue}

Several parameter sets have been used in the simulations to investigate the influence of the damage parameters over the microsphere model. We have imposed a homogeneous deformation gradient as $\mathbf{F}=1 / \sqrt{\lambda} \mathbf{e}_{x} \otimes$ $\mathbf{e}_{x}+1 / \sqrt{\lambda} \mathbf{e}_{y} \otimes \mathbf{e}_{y}+\lambda \mathbf{e}_{z} \otimes \mathbf{e}_{z}$. These simulations have been carried out up to $\lambda=3$. The mean direction of 
the fiber is placed along $\mathbf{e}_{z}$. Table 1 presents the different sets of parameters for each case while Fig. 3 shows a graphic representation of the macroscopic behavior of the material. All the results in Fig. 3a, b and c were obtained using 368 integration directions. Moreover, we present Online Resource 1, 2 and 3 (reduction factor, stress without damage and with damage respectively) to show the evolution of the micro-structure along the load in the stereographic projection (see Miehe et al. (2004); Alastrué et al. (2009a)), for the parameters of Set 1 .

\begin{tabular}{cccccc}
\hline & $k_{1}[\mathrm{kPa}]$ & $k_{2}[-]$ & $a[-]$ & $c[\mathrm{kPa}]$ & $\mathrm{b}[-]$ \\
\hline Set 1 & 100 & 1 & 1 & 50 & 1 \\
Set 2 & 100 & 1 & 0.1 & 50 & 1 \\
Set 3 & 100 & 1 & 1 & 30 & 1 \\
Set 4 & 100 & 1 & 1 & 50 & 10 \\
\hline
\end{tabular}

Table 1: Parameter sets for the displacement driven uniaxial test

Variation of parameter a. As shown in Fig. 2, the parameter $a$ controls the evolution of the damage evolving faster when $a$ increases. The evolution of the stress over the stretching range is presented in Fig. 3a. A smoother response for $a=0.1$ than for $a=1.0$ can be observed.

Variation of parameter $c$. The material parameter $c$ represents the value of the energy density function at which the damage reaches $50 \%$. The lower the value of $c$ the lower the value of the stress achieved due to the earlier failure of the fibers. This fact is captured in Fig. $3 \mathrm{~b}$ where the $c=30 \mathrm{kPa}$ curve presents a lower stress than the $c=50 \mathrm{kPa}$ one.

Variation of parameter $b$. Fig. $3 c$ shows the influence of $b$ in the model. Higher concentrations of the fibers cause a faster failure of the material. As soon as the integration directions within the influence area of the statistical distribution fails there are not other fibrils to carry load.

Variation of the number of integration directions. To conclude with this section, 78, 368 and 18476 integration directions (ID) have been used with the parameter set 1 , and the results are shown in Fig. 3d. The two first schemes come from the cubatures providing high order approximations (Heo and Xu, 2001). The 18476 integration direction scheme was obtained by a finite element mesh of triangular elements over the surface of the unit sphere. The integration directions are the centroids of the element while the weights are calculated by means of an area associated to each element. As the number of directions increases, the number of peaks also decreases and for ID $\mapsto \infty$ the curve tends to a smoother response without peaks. Therefore, an increase of the integration directions leads to more accurate results. Another possible choice would be to consider another integration technique such as that used by Hardin and Sloane (1996) or the non-linear transformation, proposed by Alastrué et al. (2009b), in order to adjust the distribution of the integration directions to the statistical distribution function. The discretization and associated peaks may be considered as lacking accuracy. However, in a recent work, Tang et al. (2010) presented some results realting to the deformation of collagen fibrils under uniaxial tension where, as the fibril stretched, it started to fail showing a behavior very similar to that presented in this section, demonstrating that a finite number of fibrils in each fiber bundle could lead to a more realistic behavior of fibers than that achieved by an ideally perfect integration scheme for an affine scenario.

\subsection{Thin perforated plate: Macro-behavior}

A thin perforated plate is a widely used critical benchmark problem to check convergence and results in plasticity and damage models (Miehe, 1995; Souza Neto et al., 1998). Different parameter sets were also analyzed for this geometry. The model consists of an eighth part of the whole geometry applying symmetry conditions (symmetry on the three planes) and pulling 15 $\mathrm{mm}$ along $\mathbf{e}_{x}$, as shown in Fig. 4a. The finite element mesh consists of 200 hexaedral elements. We performed several simulations with refined meshes, showing the classical localization problem. We have included one family of fibers, denoted by $\mathbf{m}_{0}(4 \mathrm{a})$. For this particular example we have oriented the fiber in $\mathbf{e}_{x}$. In order to visualize the micro-stresses and micro-damage in the model, a stereographic projection has been used on the integration point of the finite element highlighted in Fig. 4b. The parameters selected and the maximum convergence level are shown in Fig. 2.

\begin{tabular}{ccccccc}
\hline & $k_{1}[\mathrm{kPa}]$ & $k_{2}[-]$ & $a[-]$ & $c[\mathrm{kPa}]$ & $b[-]$ & $\%$ \\
\hline Case 1 & 50 & 1 & 1 & 10 & 1 & 63.8 \\
Case 2 & 50 & 1 & 1 & 5 & 1 & 100 \\
\hline
\end{tabular}

Table 2: Parameter sets for the displacement driven plate

Fig. 5 and Fig. 6 show the contour plot of the reduction factor and $\sigma_{x}$, the Cauchy stress tensor component in the $\mathrm{X}$ direction for Cases 1 and 2 defined in Table 2. Figs. $5 \mathrm{a}$ and $5 \mathrm{~d}$ present the stress field for $\sigma_{x}$ at $50 \%$ and $100 \%$ of the analysis respectively. Figs. $5 \mathrm{~b}$ and $5 \mathrm{e}$ 


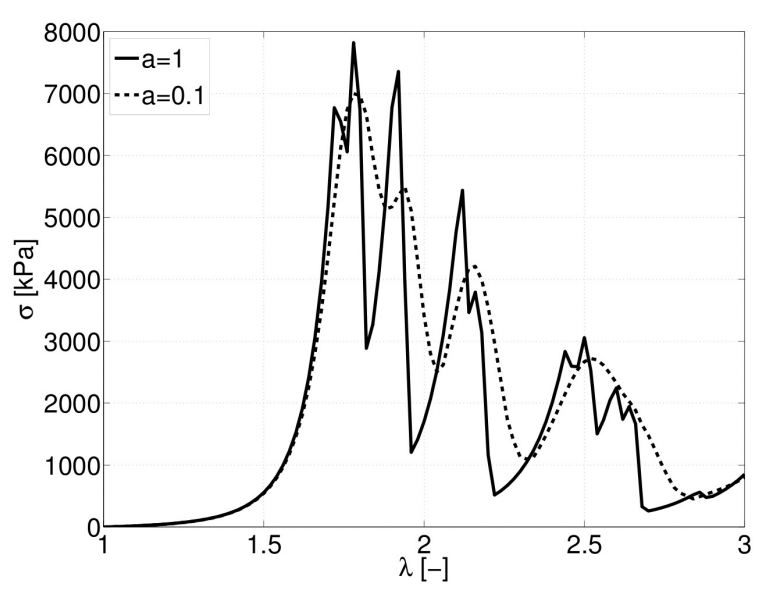

(a) Stress in the $\mathbf{e}_{z}$ direction for Set 1 and Set 2 .

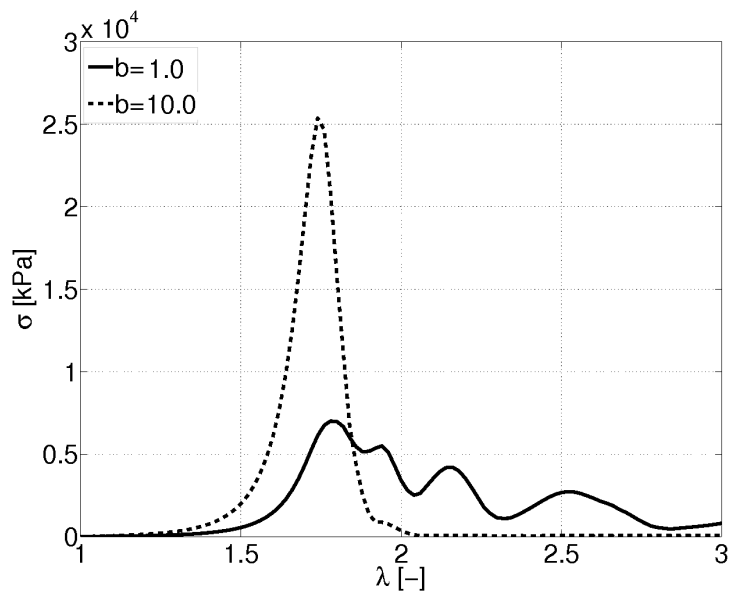

(c) Stress in the $\mathbf{e}_{z}$ direction for Set 2 and Set 4 .

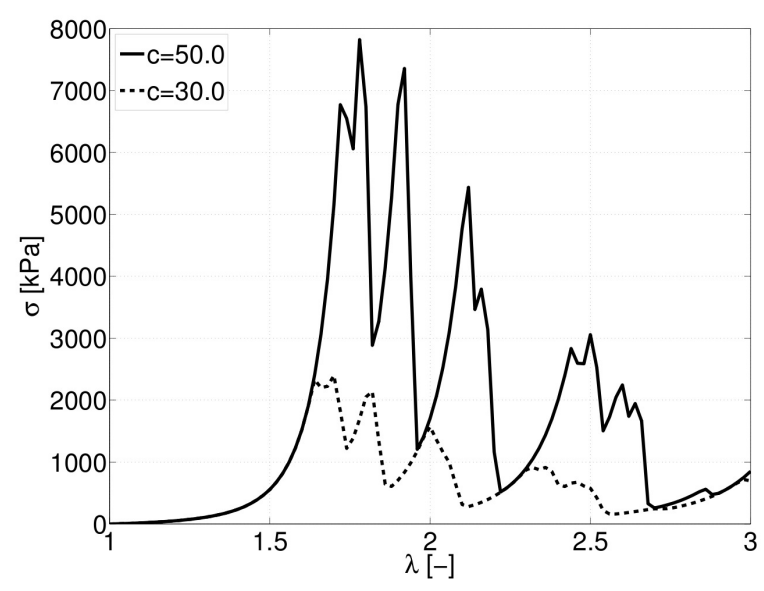

(b) Stress in the $\mathbf{e}_{z}$ direction for Set 1 and Set 3.

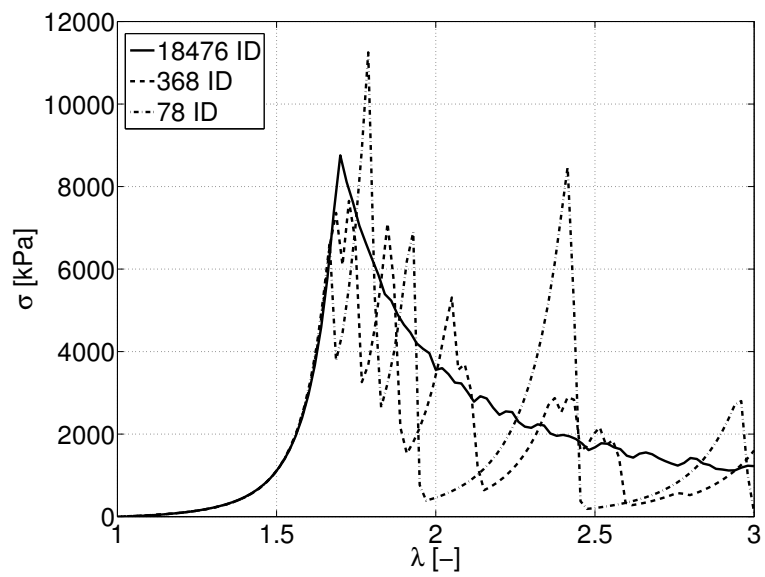

(d) Stress in the $\mathbf{e}_{z}$ direction using 78, 368 and 600 integration directions.

Fig. 3: Stress-stretch curves in the loading direction. The default parameters are $k_{1}=100[k P a], k_{2}=1, b=1$, $a=1$ and $c=50$. (a) results for several values of $a$, (b) of $c$ and (c) of $b$. (d) results for 78, 368 and 18476 integration directions using Set 1.

show the reduction factor $g$ at both strain levels while Figs. $5 \mathrm{c}$ and $5 \mathrm{f}$ present the micro stresses $\sigma_{x}$. Similar figures for Case 2 are shown in Fig. 6 .

Some important differences can be remarked in both cases. Case 1 presents a stiffer response, since the damage starts later than in Case 2, while total damage is not achieved in the former. At $50 \%$ of the analysis Case 1 presents a maximum reduction factor of $g=0.84$ and at the end reaches 0.6 along the integration directions most aligned with the $\mathbf{e}_{x}$ axis (note that when $g$ decreases, the damage $\mathrm{D}$ increases). Case 2, with damage starting sooner, showed a reduction factor of $g=0.9$ at $50 \%$ with a more spread distribution and a damage close to 1 is obtained at $100 \%$ of strain. It is worth noting the evolution of the stress over the stereographic projection. Fig. $6 \mathrm{~b}$ makes clear that the fibers along the $\mathbf{e}_{x}$ axis present a higher value of stress (although lower than that achieved without damage). Fig. 6e shows a crown-like shape caused by the total failure of these fibrils oriented along $\mathbf{e}_{x}$. Moreover, the higher the achieved strains, the higher the displacement of the crown towards the middle plane.

\section{Application to blood vessels}

One of the aims of these models is the application to soft biological tissue and the realistic simulation of clinical applications. Angioplasty is one of the most widely used techniques in vascular surgery. Therefore, the study of the mechanical response of vessels in this situation is an important task since this procedure can affect the vessel 


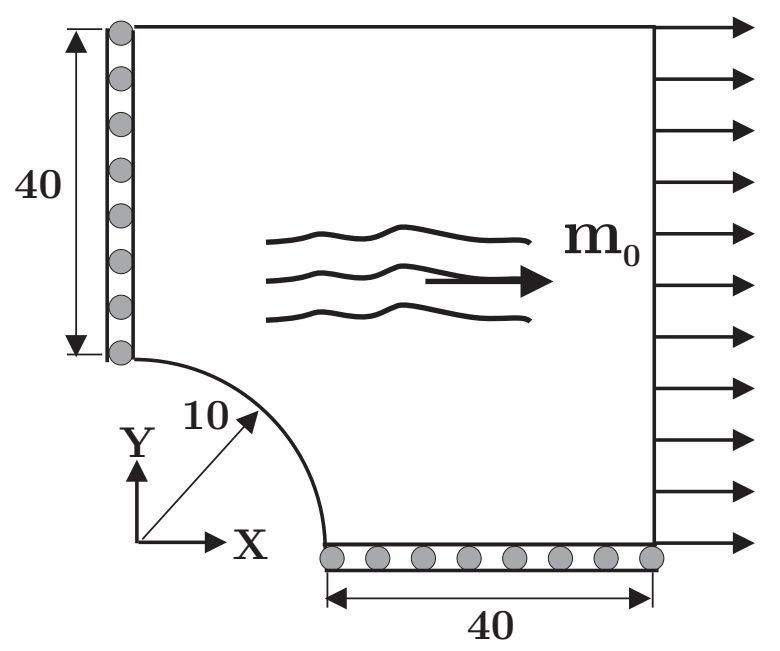

(a) Boundary conditions.

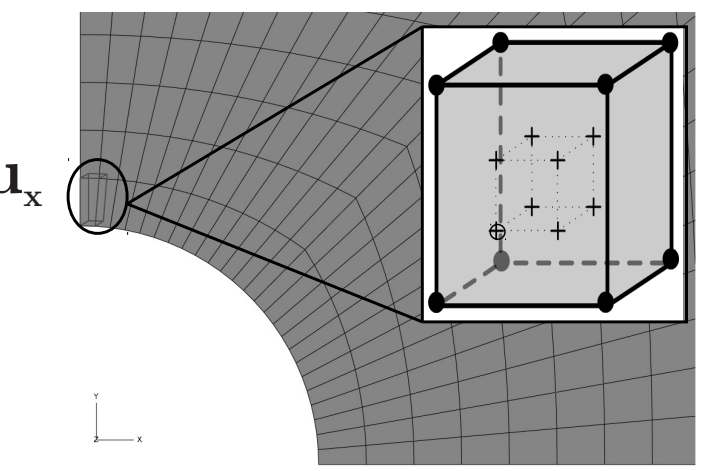

(b) Finite element mesh, element and integration point chosen for plotting results.

Fig. 4: Geometry and mesh for the thin perforated plate problem.

integrity reducing its stiffness (Oktay, 1994). In order to assess this behavior, an angioplasty procedure was simulated using the above presented model. A pig aorta artery was experimentally tested to fit the elastic and damage material parameters of the model which were subsequently used in the finite element simulations.

\subsection{Parameter identification}

In the following section the material parameters of the constitutive behavior and the damage model are fitted using experimental data (Peña et al., 2010). Vessel samples were cut comprising longitudinal and a circumferential strips in order to perform uniaxial tests. A least-square method was used for the identification process. The fitting procedure was carried out in two steps, the first for the elastic part (before damage) and the second for the damage response, when the softening phenomenon has started. The objective function to minimize was

$\mathcal{X}^{2}=\sum_{j=1}^{p}\left[\left[\sigma_{\theta \theta}-\sigma_{\theta \theta}^{\Psi}\right]_{\mathrm{j}}^{2}+\left[\sigma_{\mathrm{zz}}-\sigma_{\mathrm{zz}}^{\Psi}\right]_{\mathrm{j}}^{2}\right]$

where $p$ is the number of points taken from the experimental test, $\sigma_{\theta \theta}$ and $\sigma_{\mathrm{zz}}$ are the Cauchy stress experimental data, $\sigma_{\theta \theta}^{\Psi}$ and $\sigma_{z z}^{\Psi}$ the model derived Cauchy stress $\left(\boldsymbol{\sigma}^{\Psi}=J^{-1} \boldsymbol{\tau}^{\Psi}\right)$. Moreover, to quantify the goodness of the fitting, the root mean square error $\varepsilon$ (NRMSE) was used.

$\varepsilon=\frac{1}{\nu} \sqrt{\frac{\mathcal{X}^{2}}{[p-q]}}$ with $\nu=\frac{1}{p} \sum_{j=1}^{p}\left[\sigma_{\theta \theta}-\sigma_{z z}\right]$, with $q$ the number of parameters to be identified and $\nu$ the mean stress. Holzapfel et al. (2005) reported that choosing a right length-width ratio ensures a homogeneous state of deformation in the measuring area. In this approach, incompressible behavior has been considered with a deformation gradient tensor given by $\mathbf{F}=1 / \sqrt{\lambda} \mathbf{e}_{x} \otimes \mathbf{e}_{x}+1 / \sqrt{\lambda} \mathbf{e}_{y} \otimes \mathbf{e}_{y}+\lambda \mathbf{e}_{z} \otimes \mathbf{e}_{z}$, considering strain in the direction of pulling: $\mathbf{e}_{\theta}$ for the circumferential sample; or $\mathbf{e}_{z}$ in the longitudinal one. Following these assumptions, Table 3 shows the identified parameters for the experimental test performed by Peña et al. (2010). Note that $\alpha$ represent the angle of the fibers with respect to the circumferential direction. Fig. 7 presents the curves corresponding to the experimental data and those derived from the fitted parameters. In order to fit the experimental tests we have used a homogeneous state which corresponds to the state presented in Section 3.1. This is not the real situation since, as discussed above, it is related to the micro-structure. The optimum situation would be to fit such experimental tests with a non-uniform geometric phantom, in order to impose inhomogeneous states different for all over the phantom. However, this leads to the classical convergence problems making the fitting process difficult. 


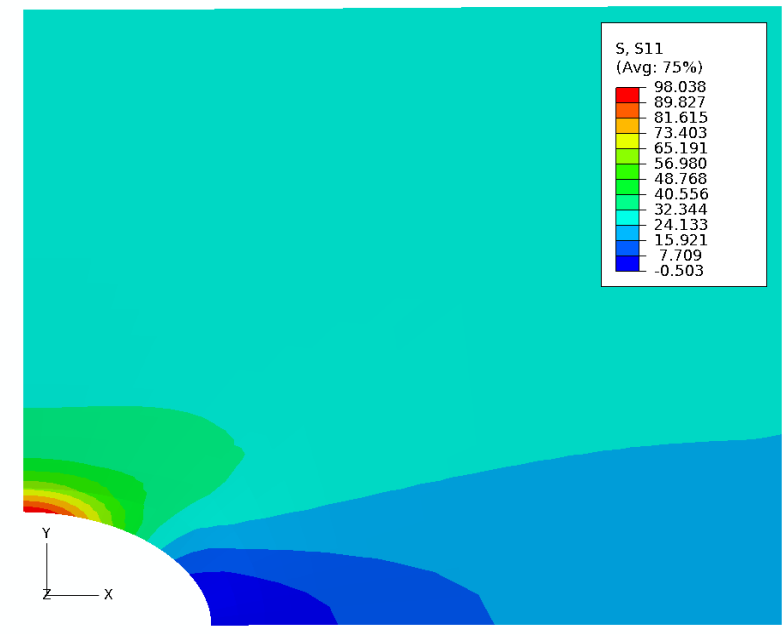

(a) Cauchy stress field $[\mathrm{kPa}]$ in the $\mathrm{X}$ axis at $36.9 \%$ of the analysis.

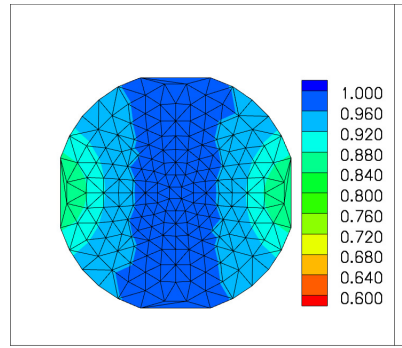

(b) $g$ at $36.9 \%$.

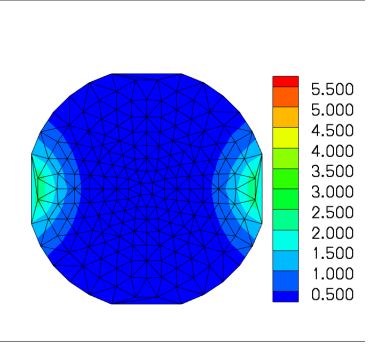

(c) $\sigma_{x}$ at $36.9 \%$

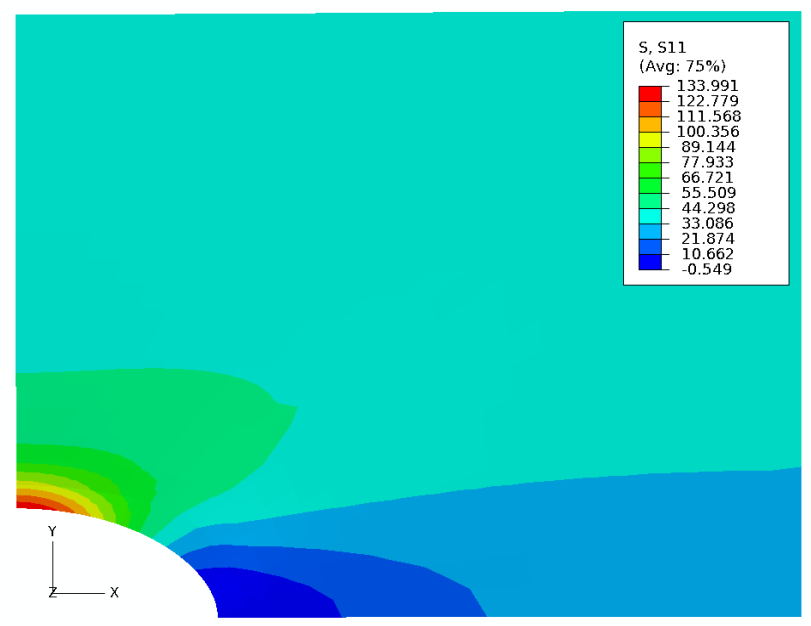

(d) Cauchy stress field $[\mathrm{kPa}]$ in the $\mathrm{X}$ axis at $63.8 \%$ of the analysis.

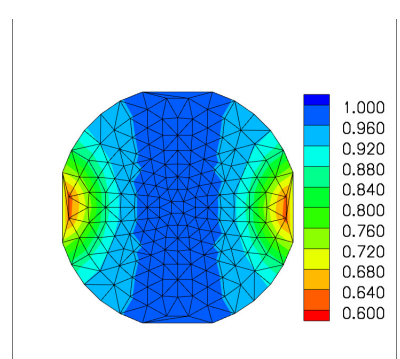

(e) $g$ at $63.8 \%$.

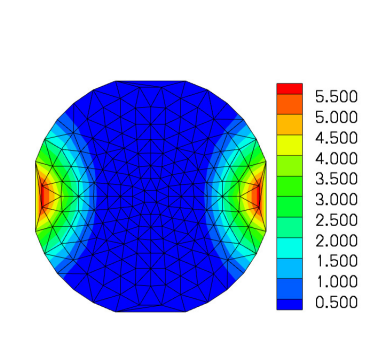

(f) $\sigma_{x}$ at $63.8 \%$.
Fig. 5: Evolution of stress and reduction factor at the integration point shown in Fig. 4b for Case 1.

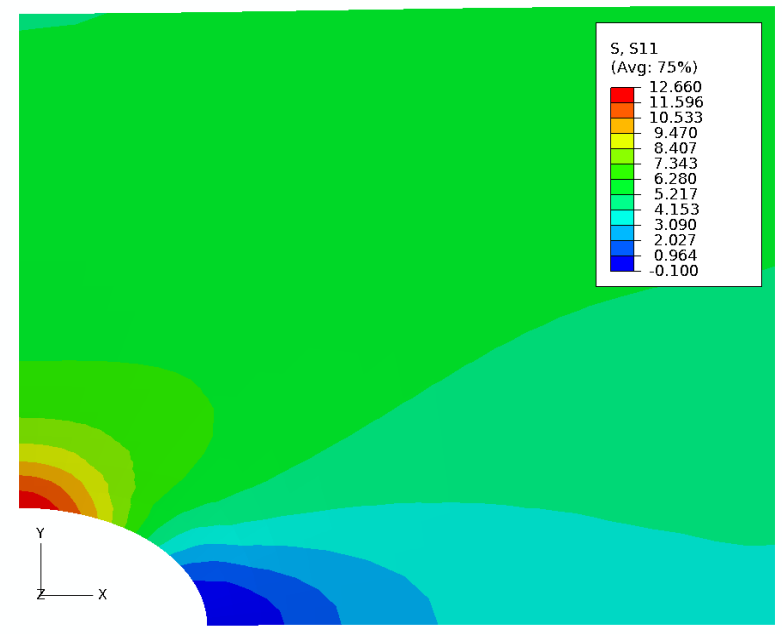

(a) Cauchy stress field $[\mathrm{kPa}]$ in the $\mathrm{X}$ axis at $50 \%$ of the analysis.

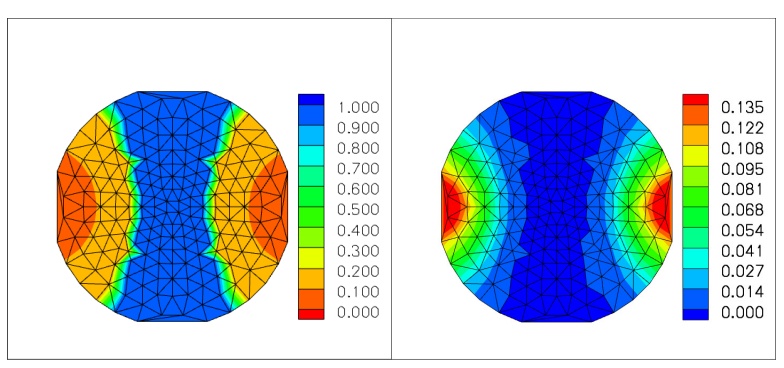

(b) $g$ at $50 \%$

(c) $\sigma_{x}$ at $50 \%$.

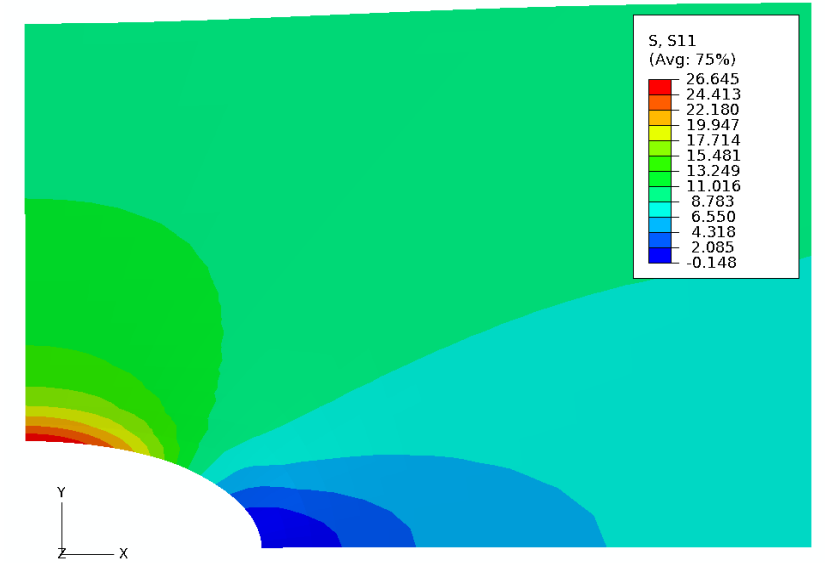

(d) Cauchy stress field $[\mathrm{kPa}]$ in the $\mathrm{X}$ axis at $100 \%$ of the analysis.

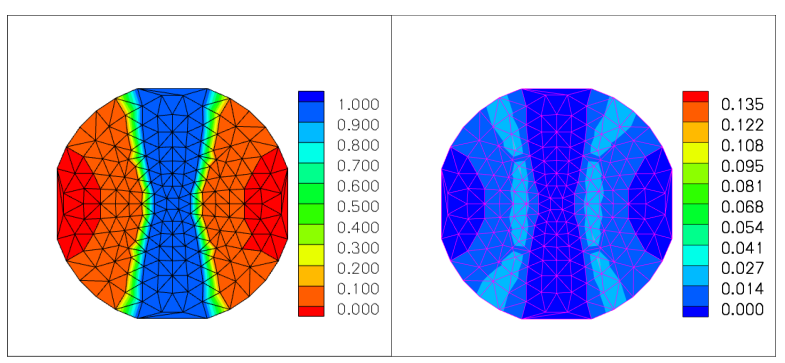

(e) $g$ at $100 \%$.

(f) $\sigma_{x}$ at $100 \%$.

Fig. 6: Evolution of stress and reduction factor at the integration point shown in Fig. 4b for Case 2. 


\begin{tabular}{ccccccc}
\hline$\mu[\mathrm{kPa}]$ & $k_{1}[\mathrm{kPa}]$ & $k_{2}[-]$ & $\mathrm{b}[-]$ & $\mathrm{a}[-]$ & $\mathrm{c}[\mathrm{kPa}]$ & $\alpha[\mathrm{deg}]$ \\
\hline 1.051 & 286.36 & 1.40 & 10.62 & 0.41 & 20.10 &
\end{tabular}

Table 3: Elastic and damage parameters for the uniaxial test

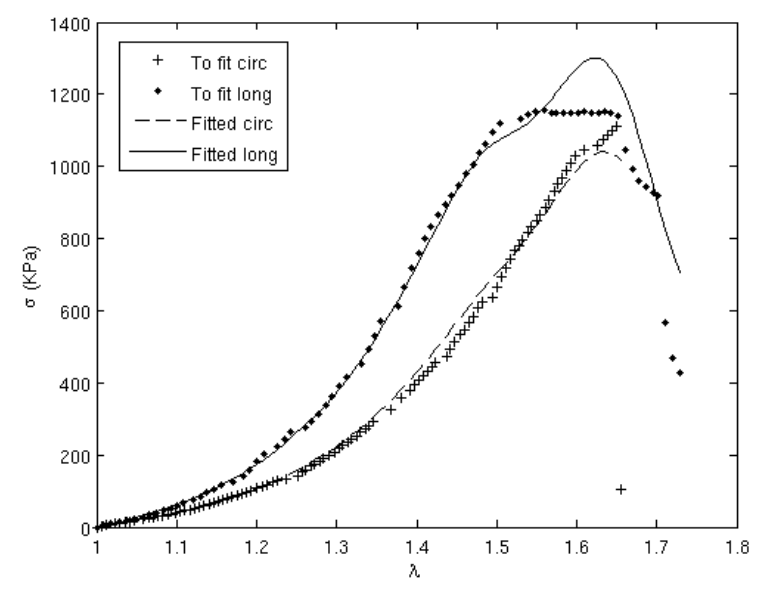

Fig. 7: Experimental and fitted curves.

\subsection{Simulation of angioplasty}

The goal of this numerical example is to show the applicability of the presented model to simulate the vessel behavior, including damage, and not the rigorous simulation of a clinical study. For that purpose, it would be necessary to take into account further aspects (e.g. a separated experimental test and simulation for each of the arterial layers should be performed). The finite element simulation of the angioplasty was performed in a eighth part of the model, applying symmetry conditions. The model consists of a $10[\mathrm{~mm}]$ length phantom with an external diameter of $D_{e}=5[\mathrm{~mm}]$ and an internal diameter of $D_{i}=3.7[\mathrm{~mm}]$ corresponding to dimensions of coronary arteries. One layer only was considered since no experimental data were available for the separate layers. The fiber angle with respect to the circumferential direction together with the parameters are shown in Table 3 . The artery geometry was discretized in 5500 hexahedral elements. Balloon dimensions and material properties were previously reported in Alastrué et al. (2007b) and Gasser and Holzapfel (2007). The load steps were applied sequentially as follows: (i) imposition of an initial deformation gradient (Alastrué et al., 2007a), (ii) application of an internal pressure of $13.3[\mathrm{kPa}]$ in the vessel assuming this as the average physiological hemodynamic pressure and (iii) imposition of pressure to the internal face of the balloon fol- lowing the curve shown in Alastrué et al. (2007b) in order to achieve contact between vessel and balloon. The undeformed and deformed configurations of the model are presented in Fig. 8a and $8 \mathrm{~b}$ respectively. The maximal principal Cauchy stress field on the artery at the end of the analysis is presented in Fig. 9a. The maximal stresses appear at the inner radius of the vessel due to the one layer simplification. Some other authors (Alastrué et al., 2007b) observed different distributions of stresses due to the incorporation of two different layers. In order to illustrate an average value of the damage of every integration direction at the gauss integration points of the mesh, an average reduction factor is defined as

$G_{\text {ave }}=\int_{\mathbb{U}^{2}} \rho g \mathrm{~d} A \approx \sum_{i=1}^{m} \rho_{i} g_{i} w^{i}$.

In agreement with the stress results, the Fig. 9b shows that the highest damage value is localized at the inner radius. Fig. 10 presents the circumferential stress, showing that the vessel starts to suffer damage in the last increments of the analysis where a clear softening effect appears. Moreover, we present the damage field in the integration direction aligned with $\mathbf{e}_{x}$ (Fig. 11a), the averaged damage field (Fig. 11b), maximum stress field (Fig. 11c) and minimum stress field (Fig. 11d) in the plane of axial symmetry imposition. Fig 11a shows an almost total failure of the fibrils aligned in $\mathbf{e}_{x}$, although the average damage does not reach such a high damage level (Fig. 11b).

\section{Conclusions}

The aim of this work is to present the complete formulation of a damage model within an anisotropic microspherebased approach in order to achieve a better characterization of this phenomenon for biological soft tissues. The model is formulated in a hyperelastic framework, using the splitting of the free Helmholtz energy density function into volumetric and isochoric parts, which was additionally decoupled into an isotropic part, associated with the ground matrix and an anisotropic part, related to the collagen fibers. To this end, the free energy density associated to the fibrils is expressed in terms of the stretches. In the framework of a multi scale model, a homogenization scheme was required to move from the micro to the macro level. A microsphere-based approach was used for this purpose, carrying out a numerical integration of the stress and elasticity tensors over the surface of the unit sphere. Moreover, within this approach, a phenomenological damage model, previously developed for unidirectional families of fibers 


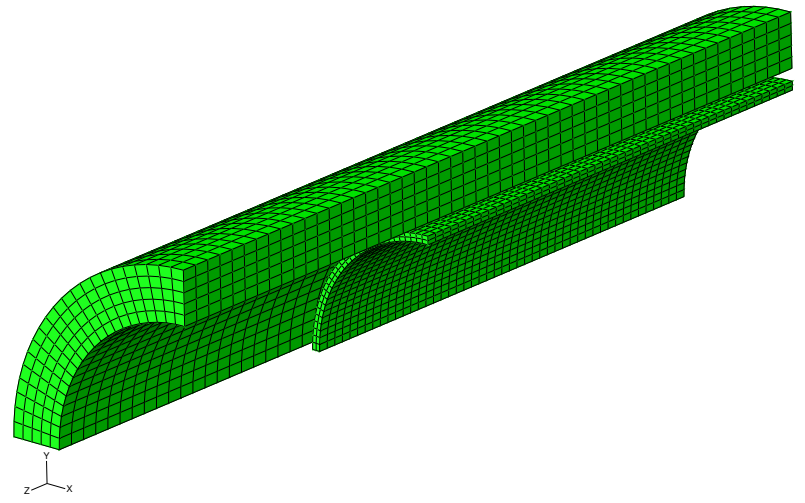

(a) Undeformed shape.

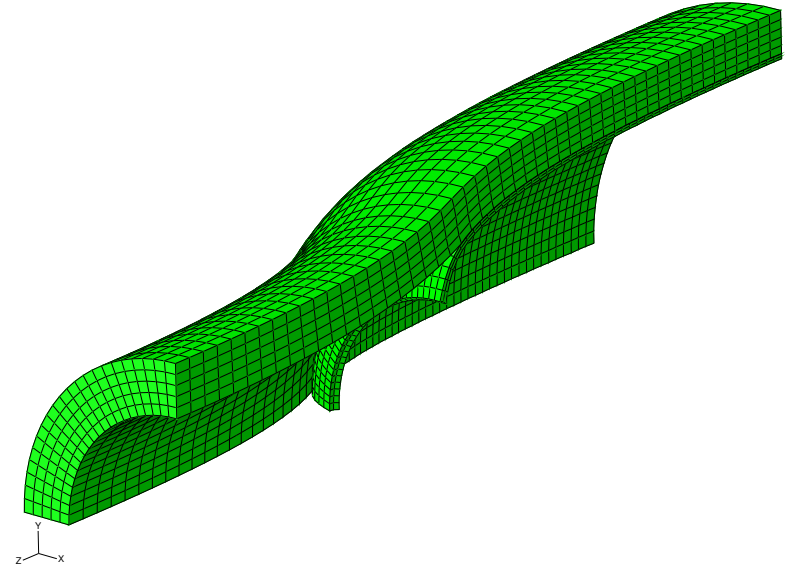

(b) Deformed shape.

Fig. 8: Undeformed and deformed shapes for the angioplasty simulation.

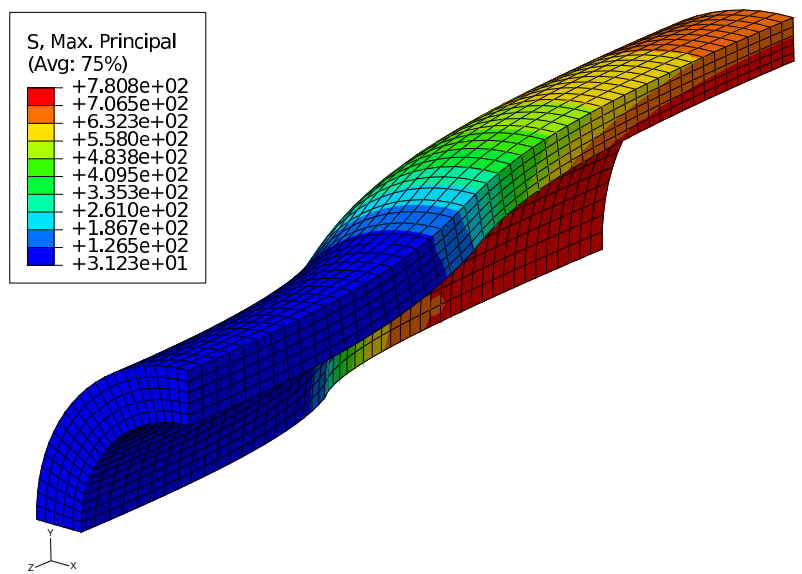

(a) Maximal principal Cauchy stress field.

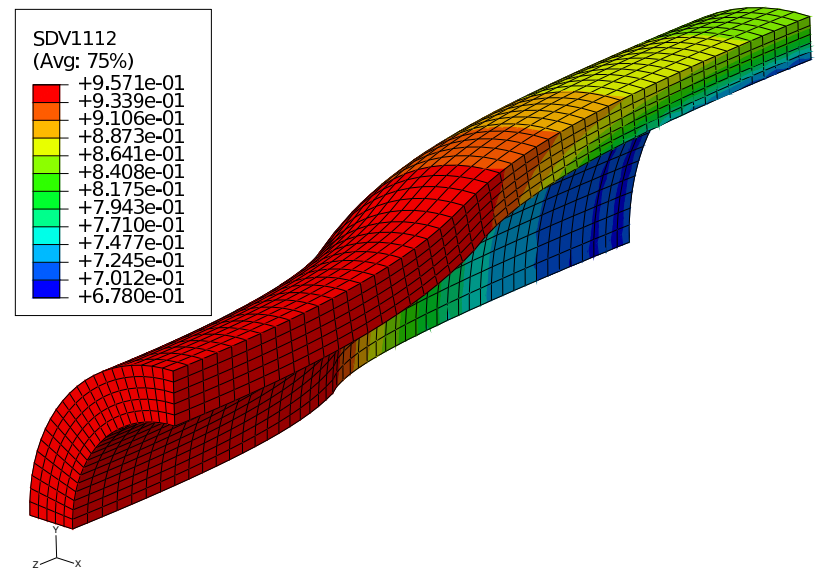

(b) Average damage field.

Fig. 9: Stress and average damage at the end of the analysis .

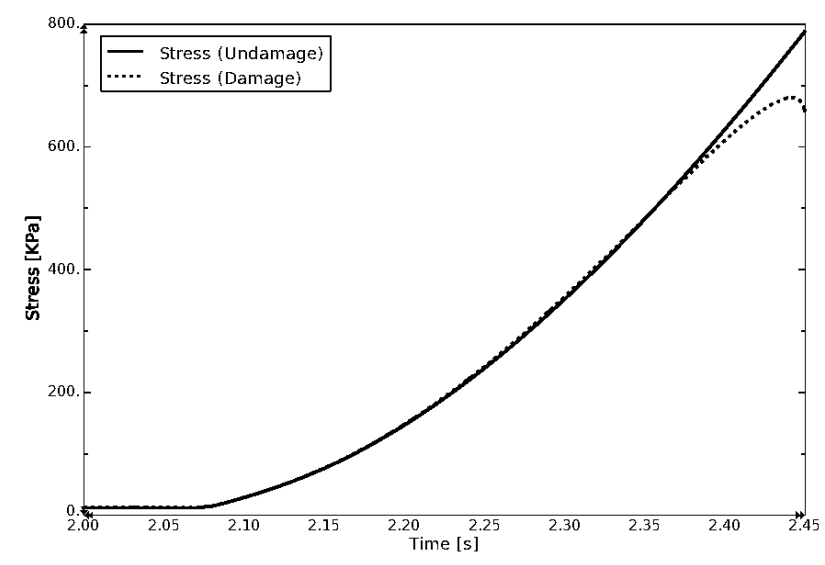

Fig. 10: Maximum Cauchy stress for circumferential direction along the damaged and undamaged model. was incorporated into the microsphere-based approach, restricted to affine deformations. Previous works (Alastrué et al., 2009a) studied different discretizations for an accurate numerical integration, leading to a 368 discretization as an optimum value in the elastic range.

The model was implemented in a commercial finite element code (ABAQUS V6.9) through a material subroutine (UMAT) and checked with different sets of material parameters and geometries. A homogeneous defomation state was imposed and the results showed some important characteristics of the proposed model. As far as the damage parameters are concerned, the lower the parameter $a$ (related to the evolution rate of the damage), the smoother the evolution of the stress; while the lower the value of $c$ (energy level at which damage starts) the lower the stress achieved. The higher 


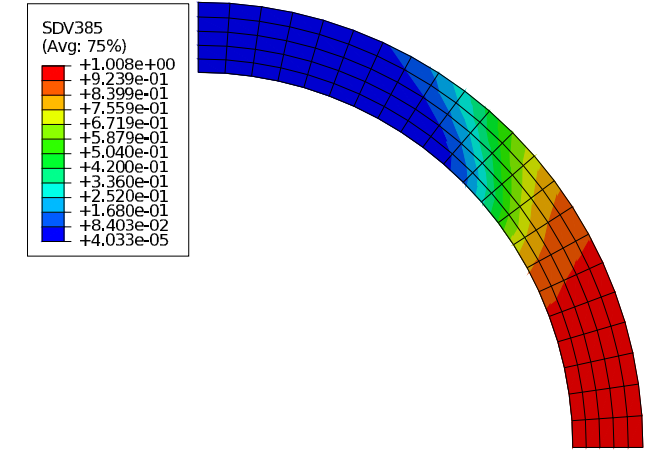

(a) Damage field for a integration direction aligned in $\mathbf{e}_{z}$.

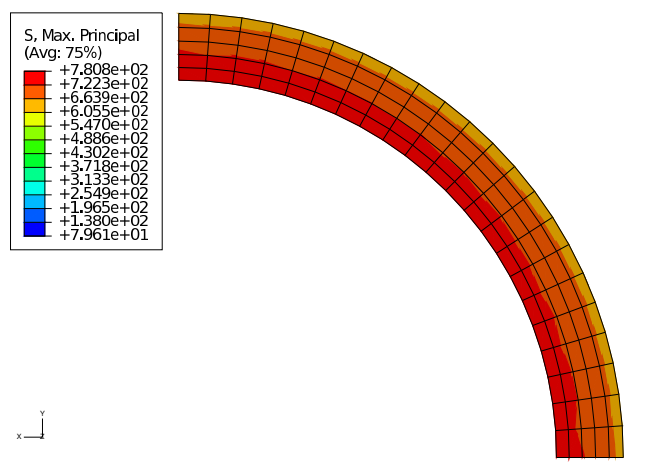

(c) Maximum principal stress field.

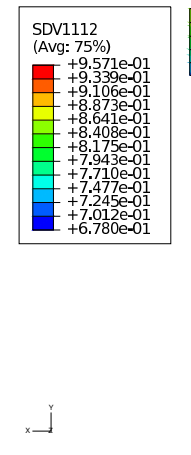

(b) Average damage field.

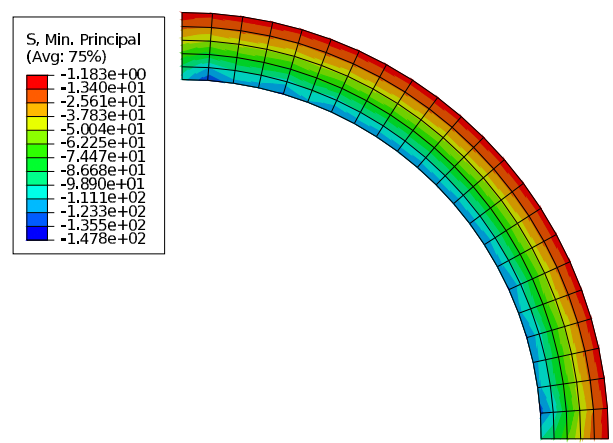

(d) Minimum principal stress field.

Fig. 11: Damage and stress fields in the axial symmetry plane.

the concentration parameter, the higher the maximum value of stress and the lower the strains needed for total failure. The integration scheme used showed that as the number of integration directions increases (18476 ID), a smoother response is achieved. However, schemes with a lower number of integration directions provide a new approach to structures where non-smooth responses were reported. In order to obtain the behavior in a non homogeneous deformation state, a thin perforated plate was investigated. Moreover, this problem represents a classical convergence benchmark due to localization and loss of ellipticity (see e.g. PijaudierCabot and Bazant (1987) and Kuhl et al. (2006)) and therefore, depending on the chosen parameters, the convergence rate increases or decreases. Apart from this issue, the results showed high levels of stress and damage concentrations around the hole. Finally, an angioplasty simulation was presented, and in spite of the lack of experimental data and the mono-layer simplification, the capability of the model to simulate clinical applications, including damage was demonstrated, obtaining a high level of information. Although the insufficient experimental data available is still a handicap and some numerical integration improvements should be included in the future, these micro-structural models represent a step forward in the inclusion of micro-structural information into macroscopic models of inelastic phenomena.

Nevertheless, the present model has some limitations. The first is the undetermined parameter fitting problem. The approach followed in this work to fit the experimental data, a least-square minimization, is known to lead to a non-unique set of parameters able to reproduce the material behavior. Although some restrictions were applied in order to reduce the solution field, a unique solution can not be ensured. The best way to reduce this underdetermination would be a direct measurement of the micro-structural parameters, through experimental tests. For example, Landuyt (2006) performed measures with polarized light microscopy in order to obtain the concentration parameters of the fibers. Moreover, the convergence problems prevent us from fitting the experiment curves with a non-uniform geometric phantom, in order to impose inhomogeneous states. Therefore, we had to fit them with the homogeneous state problem in Section 3.1, which was related to the micro-structure and not to the macroscopic behavior. Another limitation, which prevents an ideal ap- 
plication of the model is associated to the discrete integration performed that leads to a non smooth response of the micro structure and the mismatch between the statistical distribution of the concentration parameter and the uniform placing of the integration directions. In cases with highly concentrated distributions, many of the integration directions are not used in an optimal way, since they correspond to directions without fibrils. Moreover, for the damage problem only $I D \rightarrow \infty$ leads to optimal results. Alastrué et al. (2009b) presented a non-linear transformation in order to reduce the number of integration directions and increase accuracy for highly anisotropic cases. Although the discrete integration performed can give rise to disadvantages, it does produce a new view of micro-structural behavior, as pointed out previously. The individual fibril contribution presents a model of a collagen fiber bundle, and as assumed in this work, the low density of cross-links between fibrils may lead to an affine deformation state within the tissue. The last important problem arises from the well known loss of ellipticity of the damage problem. Developing a model that overcomes localization problems, such as those carried out by Kuhl and Ramm (1999), Steinmann (1999), Peerlings et al. (2001) or Peña (2011) will subject for future work.

In spite of these limitations, the one dimensional character of the constitutive equations applied at the micro-level offers huge possibilities, due to its simplicity and the possibility of incorporating other microstructural variables. Menzel and Waffenschmidt (2009) reported a work related to remodeling processes, while Göktepe and Miehe (2005) and Miehe and Göktepe (2005) developed other inelastic models on the microsphere framework for isotropic materials. Moreover, the incorporation of the von Mises ODF could allow, in a more realistic way, the development of remodeling models such as those reported by Kuhl et al. (2005) or Menzel et al. (2008), with the preferential orientation direction evolving during the simulation. The use of other ODF, such as the Bingham distribution, could lead to a more flexible description of the fiber distribution (Alastrue et al., 2010). This probabilistic function may be coupled with growing and remodeling models, accounting for the mass transference and reorientation of the fibers. This latter could be modeled, for example, by modification of the ODF parameters. Therefore, it becomes clear that the research field associated to these micro-structural models is extremely wide and can lead to substantial improvements in constitutive models of soft biological tissue.

Acknowledgements The support of the Spanish Ministry of Research and Innovation through the research project DPI2010-
20746-C03-01 and through the grant BES-2009-028593, as well as the support of the CIBER initiative, is greatly appreciated. CIBER-BBn is an initiative funded by VI National D\&D\&i plan 2008-2011, Iniciativa Ingenio 2010, Consolider Program, CIBER Actions and financed by the Instituto de Salud Carlos III with assistance from the European Regional Development Fund.

\section{References}

Alastrue, V., Saez, P., Martinez, M. A., and Doblare, M. (2010). On the use of the bingham statistical distribution in microsphere-based constitutive models for arterial tissue. Mech Res Commun, 37(8):700-706.

Alastrué, V., Martinez, M. A., Doblare, M., and Menzel, A. (2009a). Anisotropic micro-sphere-based finite elasticity applied to blood vessel modelling. J Mech Phys Solids, 57(1):178-203.

Alastrué, V., Martinez, M. A., Menzel, A., and Doblare, M. (2009b). On the use of non-linear transformations for the evaluation of anisotropic rotationally symmetric directional integrals. application to the stress analysis in fibred soft tissues. Intl J Numer Meth Eng, 79(4):474-504.

Alastrué, V., Peña, E., Martinez, M. A., and Doblare, M. (2007a). Assessing the use of the "opening angle method" to enforce residual stresses in patientspecific arteries. Ann Biomed Eng, 35(10):1821-1837.

Alastrué, V., Rodriguez, J., Calvo, B., and Doblare, M. (2007b). Structural damage models for fibrous biological soft tissues. Int J Solids Struct, 44(18-19):58945911.

Ateshian, G. A. (2007). On the theory of reactive mixtures for modeling biological growth. Biomech Model Mechan, 6(6):423-445.

Balzani, D., Schroder, J., and Gross, D. (2006). Simulation of discontinuous damage incorporating residual stresses in circumferentially overstretched atherosclerotic arteries. Acta Biomater, 2(6):609-618.

Bažant, P. and Oh, B. H. (1986). Efficient numerical integration on the surface of a sphere. ZAMM-Z Angew Math Comput Me, 66(1):37-49.

Boehler, J. P. (1987). Applications of Tensor Functions in Solid Mechanics. CISM Courses and Lectures. Springer-Verlag.

Buehler, M. J. (2008). Nanomechanics of collagen fibrils under varying cross-link densities: Atomistic and continuum studies. J Mech Behav Biomed Mater, 1(1):59-67.

Calvo, B., Peña, E., Martinez, M. A., and Doblaré, M. (2007). An uncoupled directional damage model for fibred biological soft tissues. formulation and computational aspects. Int J Numer Meth Eng, 69(10):2036-2057. 
Caner, F. C. and Carol, I. (2006). Microplane constitutive model and computational framework for blood vessel tissue. J Biomech Eng, 128(3):419-427.

Carew, T. E., Vaishnav, R. N., and Patel, D. J. (1968). Compressibility of the arterial wall. Circ Res, 23(1):61-68.

Chuong, C. J. and Fung, Y. C. (1984). Compressibility and constitutive equation of arterial wall in radial compression experiments. J Biomech, 17(1):35-40.

Dal, H. and Kaliske, M. (2009). A micro-continuummechanical material model for failure of rubber-like materials: Application to ageing-induced fracturing. $J$ Mech Phys Solids, 57(8):1340-1356.

Demiray, H., Weizsacker, H. W., Pascale, K., and Erbay, H. (1988). A stress-strain relation for a rat abdominal aorta. J Biomech, 21(5):369-374.

Ehret, A. E. and Itskov, M. (2009). Modeling of anisotropic softening phenomena: Application to soft biological tissues. Int $J$ Plasticity, 25(5):901-919.

Ehret, A. E., Itskov, M., and Schmid, H. (2010). Numerical integration on the sphere and its effect on the material symmetry of constitutive equations-a comparative study. Intl J Numer Meth Eng, 81(2):189206.

Flory, P. J. (1961). Thermodynamic relations for high elastic materials. T Faraday Soc, 57:829-838.

Fung, Y. C. (1990). Biomechanics: Mechanical Properties of Living Tissues. Springer.

Gasser, T. C. and Holzapfel, G. A. (2007). Finite element modeling of balloon angioplasty by considering overstretch of remnant non-diseased tissues in lesions. Comput Mech, 40(1):47-60.

Gasser, T. C., Ogden, R. W., and Holzapfel, G. A. (2006). Hyperelastic modelling of arterial layers with distributed collagen fibre orientations. J Roy Soc Interface, 3:15-35.

Göktepe, S. and Miehe, C. (2005). A micro-macro approach to rubber-like materials. part iii: The microsphere model of anisotropic mullins-type damage. $J$ Mech Phys Solids, 53(10):2259-2283.

Hardin, R. H. and Sloane, N. J. A. (1996). Mclaren's improved snub cube and other new spherical designs in three dimensions. Discrete Comput Biol Med Geom, 15(4):429-441.

Head, D. A., Levine, A. J., and MacKintosh, F. C. (2003). Distinct regimes of elastic response and deformation modes of cross-linked cytoskeletal and semiflexible polymer networks. Phys Rev E, 68(6):061907.

Heo, S. and Xu, Y. (2001). Constructing fully symmetric cubature formulae for the sphere. Math Comput, 70:269-279.

Himpel, G., Menzel, A., Kuhl, E., and Steinmann, P. (2008). Time-dependent fibre reorientation of trans- versely isotropic continua . finite element formulation and consistent linearization. Intl J Numer Meth Eng, 73(10):1413-1433.

Holzapfel, G. A. (2000). Nonlinear Solid Mechanics: A Continuum Approach for Engineering. John Wiley \& Sons.

Holzapfel, G. A., Gasser, T. C., and Ogden, R. W. (2000). A new constitutive framework for arterial wall mechanics and a comparative study of material models. J Elasticity, 61(1):1-48.

Holzapfel, G. A., Sommer, G., Gasser, C. T., and Regitnig, P. (2005). Determination of layer-specific mechanical properties of human coronary arteries with nonatherosclerotic intimal thickening and related constitutive modeling. Am J Physiol Heart Circ Physiol, 289(5):H2048-2058.

Humphrey, J. D. (1995). Mechanics of the arterial wall: Review and directions. Crit. Rev. Bio. Eng., 23(12):1-162.

Humphrey, J. D. and Rajagopal, K. R. (2002). A constrained mixture model for growth and remodeling of soft tissues. Math Models Methods Appl Sci, 12(3):407-430.

Kuhl, E., Askes, H., and Steinmann, P. (2006). An illustration of the equivalence of the loss of ellipticity conditions in spatial and material settings of hyperelasticity. Eur J Mech A Solids, 25(2):199-214.

Kuhl, E., Garikipati, K., Arruda, E. M., and Grosh, K. (2005). Remodeling of biological tissue: Mechanically induced reorientation of a transversely isotropic chain network. J Mech Phys Solids, 53(7):1552-1573.

Kuhl, E. and Holzapfel, G. (2007). A continuum model for remodeling in living structures. J Mater Sci, 42(21):8811-8823.

Kuhl, E. and Ramm, E. (1999). Simulation of strain localization with gradient enhanced damage models. Comput Mater Sci, 16(1-4):176-185.

Landuyt, M. (2006). Structural quantification of collagen fibers in abdominal aortic aneurysms. Master's thesis, Royal Institute of Technology in Stockholm, Department of Solid Mechanics and Ghent University, Department of Civil Engineering.

Marsden, J. E. and Hughes, T. J. R. (1994). Mathematical Foundations of Elasticity. Dover Publications.

Menzel, A., Harrysson, M., and Ristinmaa, M. (2008). Towards an orientation-distribution-based multiscale approach for remodelling biological tissues. Comput Meth Biomech Biomed Eng, 11(5):505-524.

Menzel, A. and Steinmann, P. (2003). A view on anisotropic finite hyper-elasticity. Eur $J$ Mech A/Solids, 22(1):71-87.

Menzel, A. and Waffenschmidt, T. (2009). A microsphere-based remodelling formulation for 
anisotropic biological tissues. Phil Trans $R$ Soc A, 367(1902):3499-3523.

Miehe, C. (1995). Discontinuous and continuous damage evolution in ogden-type large-strain elasticmaterials. Eur. J. Mech. A. Solids, 14(5):697-720.

Miehe, C. and Göktepe, S. (2005). A micro-macro approach to rubber-like materials. part ii: The microsphere model of finite rubber viscoelasticity. J Mech Phys Solids, 53(10):2231-2258.

Miehe, C., Göktepe, S., and Lulei, F. (2004). A micromacro approach to rubber-like materials-part i: the non-affine micro-sphere model of rubber elasticity. $J$ Mech Phys Solids, 52(11):2617-2660.

Natali, A. N., Pavan, P. G., Carniel, E. L., Lucisano, M. E., and Taglialavoro, G. (2005). Anisotropic elasto-damage constitutive model for the biomechanical analysis of tendons. Med. Eng. Phys., 27(3):209214.

Oktay, H. (1994). Continuum damage mechanics of ballon angioplasty. In Internal Report. UMI.

Peerlings, R. H. J., Geers, M. G. D., de Borst, R., and Brekelmans, W. A. M. (2001). A critical comparison of nonlocal and gradient-enhanced softening continua. Int J Solids Struct, 38(44-45):7723-7746.

Peña, E. (2011). A rate dependent directional damage model for fibred materials. application to soft biological tissues. Comput Mech, page Accepted.

Peña, E., Alastrue, V., Laborda, A., Martinez, M. A., and Doblare, M. (2010). A constitutive formulation of vascular tissue mechanics including viscoelasticity and softening behaviour. J Biomech, 43(5):984-989.

Peña, E. and Doblaré, M. (2009). An anisotropic pseudo-elastic approach for modelling mullins effect in fibrous biological materials. Mech Res Commun, 36(7):784-790.

Pijaudier-Cabot, G. and Bazant, Z. P. (1987). Nonlocal damage theory. J Eng Mech, 113(10):1512-1533.

Rhodin, J. A. G. (1980). Handbook of Physiology, The Cardiovascular System, volume 2, chapter Architecture of the vessel wall, pages 1-31. American Physiological Society, Bethesda, Maryland.

Simo, J. C. (1987). On a fully three-dimensional finitestrain viscoelastic damage model: Formulation and computational aspects. Comput Method Appl M, 60(2):153-173.

Simo, J. C. and Hughes, T. J. R. (1998). Computational Inelasticity. Springer.

Souza Neto, E. A. D., Peric, D., and Owen, D. R. J. (1998). Continuum modelling and numerical simulation of material damage at finite strains. Arch. Comput. Meth. Eng., 5(4):311-384.
Spencer, A. J. M. (1971). Theory of invariants. In Continuum Physiscs, pages 239-253. Academic Press, New York.

Steinmann, P. (1999). Formulation and computation of geometrically non-linear gradient damage. Intl $J$ Numer Meth Eng, 46(5):757-779.

Taber, L. A. (1998). A model for aortic growth based on fluid shear and fiber stresses. J Biomech Eng-T ASME, 120(3):348-354.

Tang, Y., Ballarini, R., Buehler, M. J., and Eppell, S. J. (2010). Deformation micromechanisms of collagen fibrils under uniaxial tension. $J R$ Soc Interface, $7(46): 839-850$.

Truesdell, C. and Noll, W. (2004). The Non-Linear Field Theories of Mechanics. Springer-Verlag, 3rd edition.

Weisstein, E. W. (2004). "Erfi." From MathWorld-A Wolfram Web Resource. http://mathworld.wolfram.com/Erfi.html. 